\title{
A systems approach to learning, practice and reflection in emergency primary health care: Student perspectives
}

\author{
Daniel Mellifont MSportsPhysio, Nigel Barr Intensive Care Paramedic, Peter Dunn PhD \\ Affiliation: \\ Faculty of Science, Health, Education and Engineering, The University of the Sunshine Coast, Queensland, Australia
}

\begin{abstract}
Introduction

Clinical decision making skills are essential for professional practice in primary emergency healthcare and are an intended outcome of clinical and professional programs. This article documents an interdisciplinary exploration of learning and teaching of clinical decision making and critical dialectical reflection (the systems approach) at The University of the Sunshine Coast (USC), Australia.
\end{abstract}

\section{Methods}

Using an online survey we explored students' perspectives on how working with the systems approach impacted upon their confidence and capacity to engage in emergency clinical scenarios.

\section{Results}

USC's systems approach, integrated with a focus on critical reflection and case-based learning, is strongly associated with improved student confidence to engage in emergency primary health care scenarios. Most participants reported increased confidence post intervention and believed that the systems approach helped them to improve their capacity for other critical components.

\section{Conclusion}

This study indicates the beneficial nature of a systems approach to the learning and teaching of clinical decision-making. The tool could be further developed, to improve perceived ability to communicate more effectively.

\section{Keywords}

paramedic; sports medicine; transformative learning; algorithms; critical reflection

\section{INTRODUCTION}

The use of algorithms to guide emergency primary healthcare practice and improve patient safety and outcomes is well documented (8-20). Some authors advocate the use of algorithms in training (21). However, the instructional use of these frameworks to improve the acquisition of clinical reasoning and reflective practice is not particularly evident in the research literature. More recently authors have called for a move from skills-focused to a process and outcomes-based curriculum (15). Similarly, little research has been published on transformative learning through the use of such algorithmic frameworks (22).

Clinical decisions are made from a set of options that have uncertain outcomes and inherent risks (4). Clinical decisions open up a variety of outcomes that range from fundamentally bad to profoundly good (including critical life-and-death outcomes). The results of the decisions may shorten or extend life, or alter one's capacity to engage with all future opportunities that flow after the clinical decision is made. Clinicians thus 
require a framework upon which to base decisions, particularly in emergency events. Clinical decision-making is a fundamental practice developed through clinical activities in an undergraduate health science education. Accordingly, these decision processes and the uncertainties surrounding them may be leveraged by collaborating across disciplines, even those as disparate as paramedics and sport trainers, who connect through applying the continuum of primary emergency health care, with patient interests at the centre.

The knowledge, skills, and attitudes that would comprise a set of educational objectives for learning and teaching of clinical decision-making are just beginning to be defined in medicine $(1,2,4)$. Furthermore, Schwartz(1) identifies three broad domains that clinical decision-making education should encompass: the knowledge, skills, and attitudes necessary to make clinical decisions that are accurate and in the interests of the patient; the knowledge and skills required to facilitate the decisions of others whilst respecting the ethical imperative to afford informed autonomy to patients; and the understanding of the professional role and the impact on cost effective decision-making, health economics and public health guidelines.

USC's systems approach tool was developed through critical dialectical reflection. The tool combines the relative procedural strengths of both paramedics (assessment and clinical management of patients) (23) and sports trainers (e.g. assessment of non life-threatening musculoskeletal injuries according to Sports Medicine Australia's principles of management) (24). Subsequent iterative development has integrated the systems approach tool with learning and teaching practices that focus on critical reflection and case-based learning; utilising these as drivers for development in clinical reasoning.

The development of clinical decision-making skills is essential for undergraduate students seeking to embark on a career in emergency primary health care (1). While the area of clinical decision making by paramedics has attracted little research (2), paramedic knowledge and judgment practices are a complex interplay of factors contributing to the expert practitioner's capabilities (3). However, many and varied approaches to clinical decisionmaking exist $(2,4,5)$. For example, Shaban, Wyatt and Cumming (4) describe knowledgebased and rule-based approaches to decision making, and Crosskerry (6) describes a dual process theory. Even though clinical decision rules outperform individual judgement, individual judgements often override clinical rules and due process $(6,7)$.
Interdisciplinary collaboration between academics from sport science and paramedic science at the University of the Sunshine Coast (USC) identified common areas of practice with regards to clinical decision-making. A collaborative approach led to the development of a Systems Approach Tool to encourage a richer engagement in the pedagogy of clinical decision-making linked with critical dialectical reflection, which we call the systems approach (refer to Figure 1 for a copy and brief glossary of the tool). This Systems Approach Tool was developed over several iterations, in response to student, academic and industry feedback, and takes the form of a clinical algorithm. Importantly the systems approach is inextricably linked with a process of critical reflection, case-based learning and other learning experiences.

Reflective practice in clinical education demands that constructive criticism is sought, and further contributions made to the development of evidence-informed curricula and pedagogy. Accordingly, this study explores students' perspectives on how working with the systems approach impacts upon students' confidence and capacity to engage in emergency clinical scenarios. It was hypothesized that using the systems approach enhances students' confidence in:

1. engaging in emergency scenarios, and

2. their ability to put key components of emergency primary health care into practice.

\section{METHODS}

The purpose of this pilot study was to explore students' perspectives on how working with the systems approach impacted upon their confidence and capacity to engage in emergency clinical scenarios. An anonymous online questionnaire was developed as the pilot survey instrument to obtain the necessary data for analysis. The survey was self-administered by students, on a single occasion, after completion of the teaching semester. The questionnaire contained eight closed-ended and five open-ended questions eliciting both structured (Likert-style questions) and unstructured (comments and suggestions) data. Questions (see "Data collection") asked participants to reflect back on their attitudes to participating in emergency clinical scenarios before and after their use of the systems approach to inform their responses. All paramedic and sport and exercise science students at USC exposed to the systems approach tool were provided the opportunity to respond (approximately 200 students who used the tool in one second year and/or one third year undergraduate course). 
The learning design in this project had three components. The process involved:

1. Deconstruct the Systems Approach Tool by investigating the meaning of, and links between, terms

2. Reconstruct the Systems Approach Tool in a manner most meaningful to the learner. For example, cutting up and reassembling as a jigsaw, spatially reorganising the tool elements (including attaching URL's to practice guidelines) and even the reinvention of the tool as a dreamtime like narrative by an indigenous student

3. Apply the tool to scenarios across the semester. The scenarios used were both musculoskeletal and systemic health problems from the paramedic and sports science curricula.

\section{Participants}

Twenty-four out of approximately 200 students participated in this study after providing informed consent. Participants were recruited via email from undergraduate paramedic and sport and exercise science students who had used the systems approach in their respective paramedic and sports medicine courses. All 24 students answered every Likert-style question, and 14 $(58 \%)$ of these participants provided comments and suggestions.

\section{Data Collection}

Data were collected anonymously using an online survey platform (SurveyMonkey; http://surveymonkey.com).

The first section of the survey included two fourpoint Likert-style questions asking students to reflect back to both before and after they used the systems approach and report their confidence in their "ability to engage in an emergency scenario". Possible responses included Greatly lacking in confidence (GLC); Lacking in confidence (LC); Confident (C) and Very Confident (VC). Participants were also asked to explain their answers about their levels of confidence and to give reasons to justify these answers.

The second section of the survey asked participants six questions about the extent to which they agreed with statements that the systems approach helped them to improve their ability to engage in key components of first responder practice (e.g. act in a timely manner, preserve life). Possible responses were Strongly Disagree; Disagree; Agree and Strongly Agree. Participants were also invited to provide suggestions for improvement of the systems approach tool and its use for learning and practice.

\section{Statistical Analysis}

The closed-ended results from the online survey were analysed in R (25). The first research question being addressed is whether the students' self-reported confidence is the same before and after using the system tool. The null hypothesis is that the proportion of students at each level of self-reported confidence is the same before and after using the tool. Fisher's exact test was used to answer this question, due to the small numbers in the sample. The second, related research question was whether a correlation was found between students' before and after levels of selfreported confidence; the null hypothesis is that there is no correlation. Spearman's correlation was used to answer this research question, since the levels of self-reported confidence are measured on an ordinal scale.

The responses to the open-ended questions were explored and coded thematically in an iterative process (26). Given that the focus of this research is student perspectives, a constructivist approach is warranted. The four-step process described by Punch (27) was used to thematically analyse the data. This process involved, data collection, reduction of data through inferential coding, display of data and drawing conclusions. Themes were allowed to emerge from the data by using thematic coding. A priori coding based upon educational theory initially took place, and emergent coding following preliminary examination of the data was also undertaken (26). That is, an initial set of codes were developed and added to on the basis of emerging data.

\section{Ethics}

Ethical approval was granted for this study by The University of the Sunshine Coast Human Research Ethics Committee (approval number A11310).

\section{RESULTS}

Summary measures of the students' opinions before and after exposure to the tool (Figure 1) show a substantial improvement in students' overall confidence in engaging effectively in emergency scenarios. The net percentage (all confidence responses, minus all lacking confidence responses) reporting confidence before use of the tool was $42.7 \%$ (median LC); after exposure to the tool the net percentage confidence was $92.7 \%$ (median C), with only one student reporting lack of confidence. 


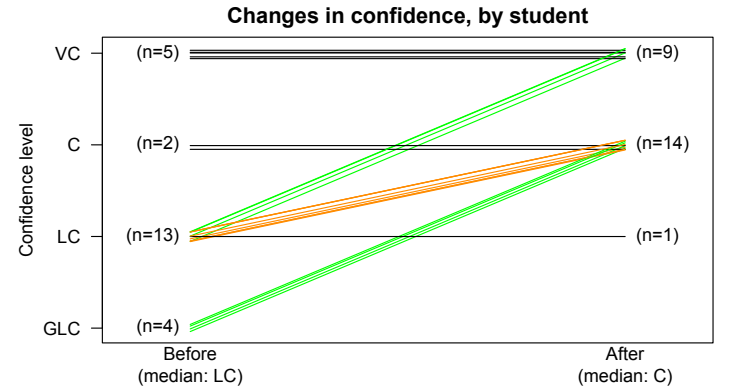

Figure 1. Student confidence before and after use of the systems approach tool

Legend: GLC means Greatly Lacked Confidence; L means lacked confidence; C means confident; VC means Very Confident.

No students reported a reduction in confidence. Of the 17 students reporting some level of lack of confidence before exposure to the tool, eight increased their confidence levels by two steps on the Likert scale, and eight by one step. Strong evidence exists of a significant relationship between students' confidence levels and when they were evaluated (before or after using the tool) (Fisher's exact test; two-tailed $p<0.0001$ ). Spearman's correlation between the before and after confidence scores shows strong evidence of a positive correlation (correlation: 0.54; two-tailed $\mathrm{p}=0.007$ ).

All 14 comments regarding confidence were consistent with two themes:

1. Before using the tool a lack of confidence was perceived and/or knowledge was not in a framework that was useful for them; and/or

2. After using the tool, students perceived an increase in their capacity to work in the field

Student comments included:

"Now that I have been equip[ped] with the systematic approach, I feel more confident that I will treat a patient more comprehensively and fluidly. It helps to give you direction in how to treat the patient and ensures "you don't miss any important assessments that could reveal something life threatening."

"The systems approach helped me to learn the important things about managing an emergency, and non emergency situation. I felt very calm and confident that I had the patient's best interests and safety in hand when dealing with an emergency scenario."

The students' confidence was also evaluated across six distinct areas of practice. The responses to individual questions concerning students' abilities (Table 1) show that the net percentage agreeing (all agreeing responses, less all disagreeing responses) was very high for all six items. The net percentage agreeing that their communication skills had improved was high at $42 \%$, but this was the lowest agreeance among the items in the survey. All responses indicated an increased capacity to apply a systems approach to care and preserve life.

Students were also invited to provide suggestions for improvement of the systems approach tool and its use. From the 32 suggestions for improvement of the systems approach tool, three clear themes emerged:

3. That the tool should be more prescriptive/segmented or more flexible/integrative;

4. That the tool needs to cater for diversity in learning styles (e.g. visual, modelling, reconstructive, compartmentalization, interactive/electronic version);

5. That the tool could be simplified.

Work is in progress to enhance the tool and its use, incorporating this feedback.

\section{DISCUSSION}

Our first hypothesis, that using the systems approach would enhance students' confidence in engaging in emergency scenarios, is supported by the data (Figure 1). Pleasingly, no student reported reduced levels of confidence after using the systems approach and the vast majority of others ( $84 \%$ of those not already "very confident) improved by at least one step (Table 1). Only three of these students reported no improved confidence (i.e. of those not already "very confident").

Secondly, we hypothesized that the tool would enhance students' abilities to put key components of emergency primary health care into practice. This hypothesis was also supported (Table 1); for example, nine respondents (37.5\%) agreed and $18(75 \%)$ strongly agreed that the system approach helped them improve their ability to act in a timely manner.

However, two interesting contrasts in results emerged. Firstly, despite results showing significant associations between use of the tool and improved confidence (Figure 1), only half (gross $71 \%$; net $42 \%$ ) of respondents felt that use of the tool helped them to improve their ability to communicate effectively (Table 1). Secondly, although a high percentage of respondents (gross $87.5 \%$, net $75 \%$,) believed that the systems approach helped them to improve their capacity for critical components of emergency first responder care (including 100\% for prevention of further injury and preservation of life), relatively fewer agreed (net $42 \%$ ) that the tool improved 


\begin{tabular}{|c|c|c|c|c|c|}
\hline Item & $\begin{array}{l}\text { Strongly } \\
\text { Disagree }\end{array}$ & Disagree & Agree & Strongly Agree & $\begin{array}{l}\text { Net count }(\%) \\
\text { agreeing }\end{array}$ \\
\hline $\begin{array}{l}\text { Apply a systems } \\
\text { approach to care }\end{array}$ & $0(0.0 \%)$ & $0(0.0 \%)$ & $14(58.3 \%)$ & $10(41.7 \%)$ & $24(100 \%)$ \\
\hline $\begin{array}{l}\text { Prioritise assessment } \\
\text { and management }\end{array}$ & $0(0.0 \%)$ & $2(8.3 \%)$ & $11(45.8 \%)$ & $11(45.8 \%)$ & $20(83 \%)$ \\
\hline $\begin{array}{l}\text { Communicate } \\
\text { effectively }\end{array}$ & $0(0.0 \%)$ & $7(29.2 \%)$ & $13(54.2 \%)$ & $4(16.7 \%)$ & $10(42 \%)$ \\
\hline Act in a timely manner & $0(0.0 \%)$ & $3(12.5 \%)$ & $12(50.0 \%)$ & $9(37.5 \%)$ & $18(75 \%)$ \\
\hline $\begin{array}{l}\text { Prevent further injury } \\
\text { (including first aid) }\end{array}$ & $0(0.0 \%)$ & $3(12.5 \%)$ & $9(37.5 \%)$ & $12(50.0 \%)$ & $18(75 \%)$ \\
\hline $\begin{array}{l}\text { Preserve life } \\
\text { (including awareness } \\
\text { of danger and } \\
\text { resuscitation) }\end{array}$ & $0(0.0 \%)$ & $0(0.0 \%)$ & $10(41.7 \%)$ & $14(58.3 \%)$ & $24(100 \%)$ \\
\hline
\end{tabular}

Table 1: The extent to which students agreed or disagreed with the statement "using the systems approach tool helped me to improve my ability to" engage in key components of first-responder practice. The net count and percentage agreeing is computed from the total number of either agreeing category, less the total number in either disagreeing category. Percentages are all row percentages of the 24 respondents.

their ability to communicate (Table 1). These results appear to indicate that the tool assists increasing students' confidence to practice. However, the ability for students to communicate their practice does not seem to be similarly improved. This may be due to limitations of the study, but perhaps communication skills are of a different domain and not adequately nurtured by the systems approach alone. This divergence of results needs to be addressed in future pedagogical practice and research.

Findings from this exploratory study provide valuable information for further development of the tool, including the possible development of an interactive (electronic) version which caters for a greater diversity of learning styles. Fourteen students provided a combined 32 suggestions for improving the tool, many suggesting a simplification. Further, results suggest a need to focus on complimentary strategies to address the importance of developing communication skills. Several opportunities for further research have been identified including deeper exploration of the relationships between use of the systems approach, confidence and clinical capacity in emergency first responders.

This study has limitations and so the results must be understood in this context. These limitations include: low participant numbers, students selfselecting into the sample, recall bias, and selfassessment of confidence levels which is not an absolute measure of clinical capacity. Although all students engaged in scenarios that focused on the care of individuals presenting with musculoskeletal and/or systemic issues, there will have been differences in the scenario details given they formed part of curricula in different programs with different scopes of practice. Furthermore, although the systems approach represents an important step on our pathway of learning and teaching improvement, we recognise that it is certainly no panacea for the challenges of clinical decision-making and may not be easily generalized to other contexts. Further research is planned to address much of these limitations (including relationship between students' perspectives and assessment of clinical capacity by impartial observers).

\section{CONCLUSION}

The System Approach Tool, integrated with a combined focus on critical reflection and casebased learning, is strongly associated with improved student confidence in engaging in emergency primary health care scenarios. Students also agree or strongly agree that use of the systems approach helps them to build their capacity to engage in key components of first responder practice, with the notable exception of communicating effectively. This exploratory pilot study indicates the possible beneficial nature of a systems approach to the learning and teaching of clinical decision-making. Furthermore, these results encourage development of decisionmaking algorithms for clinical practice and research to inform the evidence base.

\section{CONFLICT OF INTEREST}

The authors declare they have no conflict of interest. 


\section{REFERENCES}

6. Schwartz A. Medical Decision Making and Medical Education: Challenges and Opportunities. Perspectives in Biology and Medicine. 2011;54(1):68-74.

7. Shaban R. Paramedics' clinical judgment and mental health assessments in emergency contexts: Research, practice, and tools of the trade. Journal of Emergency Primary Health Care. 2006;4(2):1-13.

8. Wyatt A. Paramedic Practice - Knowledge Invested in Action. Australasian Journal of Paramedicine. 2003;1(3):Art 9.

9. Shaban RZ, Wyatt Smith CM, Cumming JJ. Uncertainty, Error and Risk in Human Clinical Judgment: Introductory Theoretical Frameworks in Paramedic Practice. Journal of Emergency Primary Health Care. 2004;2(12):1-12.

10. Anantharaman G. Standards and Standardization in Paramedic Protocols. Journal of Emergency Primary Health Care. 2004;2(1-2):1-3.

11. Croskerry $P$. Clinical cognition and diagnostic error: applications of a dual process model of reasoning. Advances in Health Sciences Education. 2009/09/01 2009;14(1):27-35.

12. Croskerry P. A Universal Model of Diagnostic Reasoning. Academic Medicine. 2009;84(8):1022-1028 1010.1097/ACM.1020b1013e3181ace1703.

13. Cayten CG, Staroscik R, Walker K, Morganroth J, Oler J. Impact of prehospital cardiac algorithms on ventricular fibrillation survival rates. Annals of Emergency Medicine. 1981;10(8):432-436.

14. Hunt RC, Bass RR, G. GR. Standing orders versus voice control. Journal of Emergency Medical Service. 1982;7:26-31.

15. Margolis CZ. Uses of Clinical Algorithms. JAMA: The Journal of the American Medical Association. February 4, 1983 1983;249(5):627-632.

16. Hoffman J, O'Neill N, Luo J. Algorithm use in the treatment of pre-hospital ventricular fibrillation: an analysis of 160 cases. Resuscitation. 1989;17:131-141.

17. Hoffman J, Luo J, Schriger D, Silver L. Does Paramedic-Base Hospital Contact Result in Beneficial Deviations From Standard Prehospital Protocols? Western Journal of Medicine. 1990;153(3):283-287.

18. Hopkins JA, Shoemaker WC, Greenfield S, Chang PC, McAuliffe T, Sproat RW. Treatment of Surgical Emergencies With and Without an Algorithm. Archives of Surgery. 1980;115(6):745-750.
19. Pointer JE, Osur MA. Effect of standing orders on field times. Annals of Emergency Medicine. 1989;18(10):1119-1121.

20. Wang HE, Kupas DF, Greenwood MJ, et al. An Algorithmic Approach to Prehospital Airway Management. Prehospital Emergency Care. 2005;9(2):145-155.

21. Armstrong B, Simpson $H$, Crouch $R$, Deakin C. Prehospital Clearance of the Cervical Spine: Does it Need to be a Pain in the Neck? Emergency medicine journal : EMJ. 2007;24(7):501-503.

22. Heard AMB, Green RJ, Eakins P. The formulation and introduction of a 'can't intubate, can't ventilate' algorithm into clinical practice. Anaesthesia. 2009;64(6):601-608.

23. Chalkias AF. Prehospital emergency thoracotomy: when to do it? Journal of Emergency Primary Health Care. 2009;7(4):112.

24. Flavell E, Boyle MJ. Which is more effective for ventilation in the prehospital setting during cardiopulmonary resuscitation, the laryngeal mask airway or the bag-valve-mask? - A review of the literature. Journal of Emergency Primary Health Care. 2010.

25. Australian Resuscitation Council. Australian Resuscitation Council: Index of Guidelines. 2012; http://www.resus.org.au. Accessed 10/1/2012, 2012.

26. Cayten CG, Oler J, Staroscik R, et al. Clinical Algorithms for Prehospital Cardiac Care. Medical Care. 1983;21(2):147-156.

27. Mezirow J. Transformative Learning as Discourse. Journal of Transformative Education. 2003;1(1):58-63.

28. Paramedics Australia. What is a Paramedic. 2013; https://www.paramedics.org/paramedics/whatis-a-paramedic/. Accessed 3/12/2013, 2013.

29. Sports Medicine Australia. Sports Medicine for Sports Trainers. 10th ed. Sydney: Mosby Elsevier; 2013.

30. R: A language and environment for statistical computing. [computer program]. Vienna, Austria: R Foundation for Statistical Computing; 2011.

31. Stemler S. An overview of content analysis. Practical assessment, research and evaluation http://pareonline.net/getvn.asp?v=7\&n=17 2001;7(17).

32. Punch K. Introduction to Research Methods in Education. London: Sage; 2009. 


\section{APPENDIX ONE}

\section{Systems Approach Tool}

\section{(C) Daniel Mellifont, Jamie Peetz, Nigel Barr \& Nick Prass}

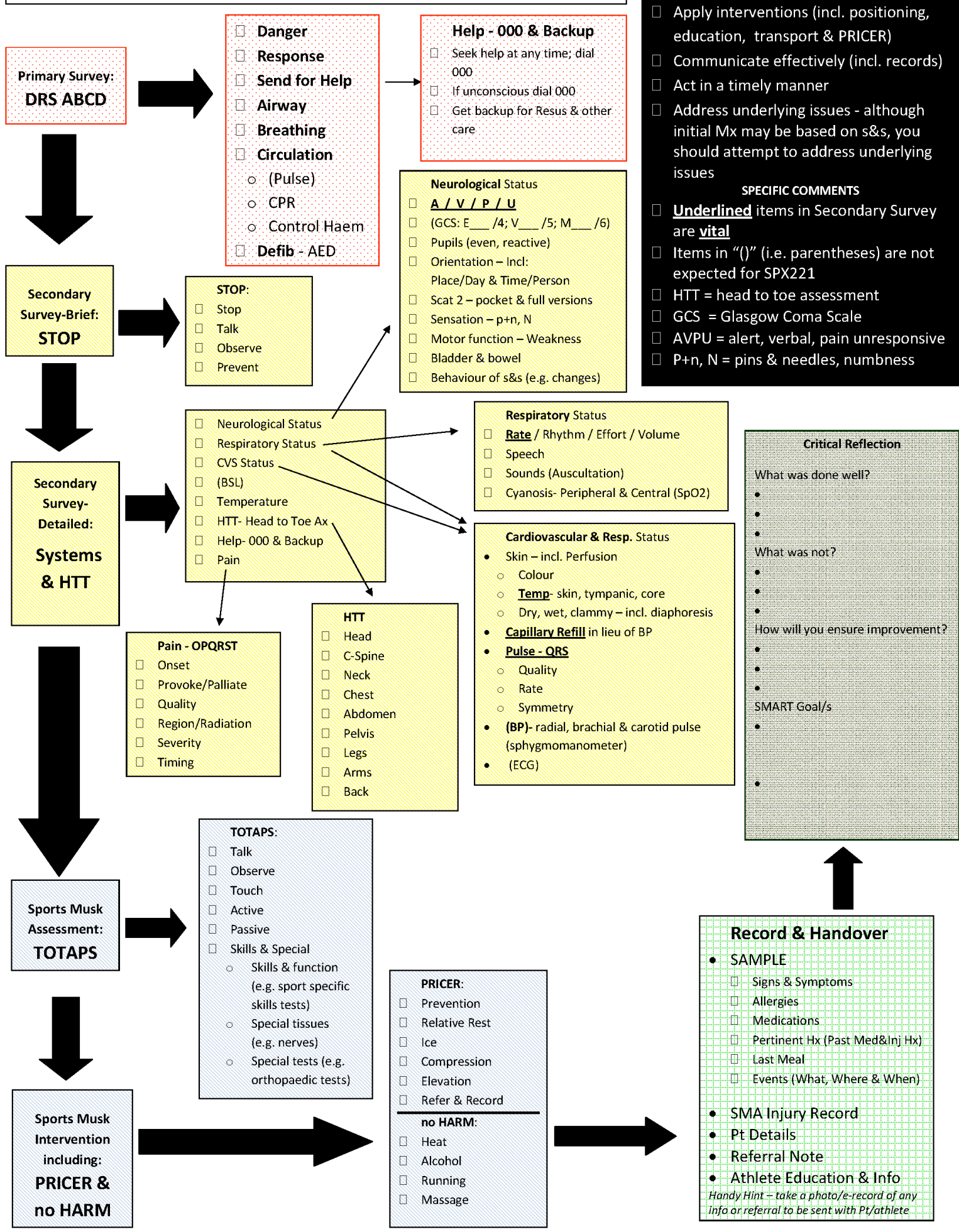

Priorities for Performance and $A x$

Preserve life (incl. DRSABCD \& Resus)

Prevent further injury (incl. $1^{\text {st }}$ Aid)

Prioritise, plan \& implement $A x \& M x$

Apply interventions (incl. positioning,

education, transport \& PRICER)

Communicate effectively (incl. records)

Underlined items in Secondary Survey

expected for SPX221

HTT = head to toe assessment

GCS = Glasgow Coma Scale

AVPU $=$ alert, verbal, pain unresponsive

$P+n, N=$ pins \& needles, numbnes
Apply the systems approach to care 


\section{APPENDIX TWO}

\begin{tabular}{ll}
\hline AED & Automated External Defibrillator \\
AVPU & Alert, Verbal, Pain, Unresponsive (a basic screen for level of consciousness by \\
BP & determining best response to stimuli) \\
BSL & Blood Pressure \\
C-Spine & Blood Sugar Level (i.e. blood glucose level) \\
CVS Status & Cervical Spine \\
DRS ABCD & Cardiovascular System Status \\
& Danger, Response, Send for help, Airway, Breathing, Circulation (Pulse, CPR, Control \\
ECG & of Haemorrhage), Defibrillation \\
GCS & Electrocardiogram \\
& Glasgow Coma Scale - E - Eye, V - Verbal, M - Motor (standardized scale to screen \\
HTT & for level of consciousness by observation and determining best response to stimuli) \\
& Head To Toe assessment (i.e. a structured approach to observation and palpation, \\
No HARM & commencing at the head and working caudally) \\
Pain - OPQRST & avoid Heat, Alcohol, Running and Massage \\
& Pain Onset, Provoking and Palliating factors, Quality, Region/Radiation, Severity and \\
p+n, N & Timing \\
PRICER & Pins and Needles, Numbness (sensation changes incl. paraesthesia) \\
Pt Details & Prevention, Relative Rest, Ice, Compression, Elevation, Refer \& Record \\
Pulse QRS & Patient Details \\
SAMPLE & Pulse Quality, Rate and Symmetry \\
& Signs \& Symptoms, Allergies, Medications, Pertinent Hx (pertinent history of injuries \\
s\&s & and medical issues), Last meal, Events \\
SCAT & Signs and Symptoms \\
SMA Injury Record & Sideline Concussion Assessment Tool (previously version 2) \\
SMART Goals & Sports Medicine Australia Injury Record form \\
SpO & Specific, Measurable, Agreed and Action oriented, Realistic and Resourced, Timely \\
STOP & and Time-bound \\
TOTAPS & Oxygen saturation levels \\
& Stop, Talk, Observe, Prevent (e.g. prevent further/harm) \\
& Talk, Observe, Touch, Active, Passive, Skills \& Special (e,g. sports specific skills \& \\
Table 2: Glossary of abbreviations contained in Figure 2
\end{tabular}

